\title{
Recent progress in the understanding of single spin asymme- tries
}

\author{
Andreas Metz ${ }^{*}$ and Marc Schlegel ${ }^{* *}$ \\ Institut für Theoretische Physik II, Ruhr-Universität Bochum, D-44780 Bochum, Germany
}

Key words Single spin asymmetries, universality, higher twist.

PACS 12.39.St, 13.60. Hb, 13.88.+e

Dedicated to Klaus Goeke on the occasion of his $60^{\text {th }}$ birthday.

Over the past years a lot of progress has been made in the understanding of single spin asymmetries in hard scattering processes. We briefly review this subject, covering the non-vanishing of time-reversal odd parton distributions, universality of fragmentation functions, and the discovery of previously unknown timereversal odd parton distributions at subleading twist. Interestingly enough, all these developments were initiated by simple model calculations.

\section{Introduction}

Non-zero single spin asymmetries (SSA) in hard scattering processes represent a very interesting phenomenon in particle physics. For instance, large asymmetries (up to $40 \%$ ) have been observed more than one decade ago in proton-proton collisions $(p \uparrow p \rightarrow \pi X)$, where one of the protons is transversely polarized [1]. (For related theoretical work see [2, 3, 4, 5] as well as [6] and references therein.) Moreover, in lepton scattering off the nucleon non-zero results for various SSA have been reported recently [7, 8]. These results are surprising because in the leading twist collinear factorization approach for hard processes SSA strictly vanish [9]. In order to get non-zero effects one has to go beyond the collinear picture and to take the transverse momentum of partons inside the hadrons into account. In this context, a special role is played by transverse momentum dependent (TMD), time-reversal odd (T-odd) correlation functions (parton distributions and fragmentation functions) which are directly connected to the SSA. Therefore, the recent progress in the understanding of SSA that has been achieved over the past three years is actually progress in the understanding of the origin and of the properties of T-odd correlation functions.

The starting point was a model calculation for inclusive deep-inelastic scattering (DIS) which has shown the importance of gluon exchange between the struck quark and the target spectators [10]. It has been demonstrated that this rescattering effect causes (additional) on-shell intermediate states in the Compton amplitude, resulting in a modification of the DIS cross section. In Feynman gauge, this (shadowing) effect is described by the gauge link appearing in the operator definiton of parton distributions.

Subsequently, the effect of rescattering has also been investigated in the case of semi-inclusive DIS [11]. Using a simple spectator model, it has been shown that a non-zero SSA for a transversely polarized target arises from the interference between the tree-level amplitude of the fragmentation process and the imaginary part of the one-loop amplitude, where the latter describes the gluon exchange between the struck quark and the target system. This asymmetry has been interpreted as model for the T-odd Sivers parton distribution [12] including its gauge link [13]. The model calculation of Ref. [11] has therefore shown for the first

\footnotetext{
* E-mail: metza@tp2.ruhr-uni-bochum.de

** E-mail: marcs@tp2.ruhr-uni-bochum.de
} 
time explicitly that T-odd parton distributions can be non-zero, which is in contrast to the long-standing believe that they should vanish because of T-invariance of the strong interaction [14]. Consequently, T-odd parton distributions may (partly) be at the origin of the experimentally observed SSA.

While T-odd parton distributions are non-zero only if the gauge link is taken into account, T-odd fragmentation functions may well exist even if the rescattering associated with their gauge link is neglected. Final state interactions can generate finite SSA in parton fragmentation [14] 15] 16]. But the presence of the gauge link endangers universality of TMD fragmentation functions, i.e., it is a non-trivial question whether the fragmentation functions in DIS and in electron-positron annihilation coincide. Whereas time-reversal can be used to relate parton distributions between DIS and the Drell Yan process [13], such an argument cannot be applied for fragmentation functions [17, 18]. However, a one-loop calculation for a SSA in fragmentation, analogous to the treatment of the target asymmetry in Ref. [11], has shown universality for T-odd fragmentation functions [19]. In a recent work it has been argued that the finding in [19] is not a model-artefact but rather a general result [18].

In [20, 21] the spectator model calculation for SSA in semi-inclusive DIS of Ref. [11] has been extended to the case of a longitudinally polarized lepton beam and a longitudinally polarized target. For both asymmetries, which are of subleading twist (twist-3), non-zero results have been found. The results have indicated the existence of previously unknown subleading twist T-odd parton distributions [21]. This conjecture has been confirmed recently by a model-independent analysis [22].

The purpose of this short review is to discuss the three mentioned developments on the field of single spin asymmetries and TMD correlation functions, i.e., (1) the non-vanishing of T-odd parton distributions, (2) the universality of TMD fragmentation functions, (3) the discovery of so far unknown T-odd parton distributions at subleading twist. Our emphasis will be on the model calculations which in all three cases preceded the model-independent studies.

\section{Gauge invariant correlation functions}

In this section we summarize certain features of parton distributions and fragmentation functions which are needed for the discussion lateron. The classical process by which parton distributions of the nucleon are measured is lepton-induced inclusive DIS, like $e^{-} p \rightarrow e^{-} X$. This reaction provides information about the leading twist parton distributions $f_{1}$ (distribution of unpolarized quarks in an unpolarized nucleon) and $g_{1}$ (distribution of longitudinally polarized quarks in a longitudinally polarized nucleon). The third twist-2 distribution, the transversity $h_{1}$ (distribution of transversely polarized quarks in a transversely polarized nucleon), cannot be extracted from inclusive DIS data because of its chiral-odd nature [23].

A proper definition of these three distributions is given in terms of the light-cone correlator

$$
\Phi_{i j}(x, S)=\int \frac{d \xi^{-}}{2 \pi} e^{i x P^{+} \xi^{-}}\left\langle P, S\left|\bar{\psi}_{j}(0) \mathcal{W}\left(0, \xi^{-}\right) \psi_{i}\left(\xi^{-}\right)\right| P, S\right\rangle .
$$

In Eq. (1) the target state is characterized by its four-momentum $P$ and the covariant spin vector $S\left(P^{2}=\right.$ $\left.M^{2}, S^{2}=-1, P \cdot S=0\right)$. The longitudinal quark momentum is specified by $x$ via $k^{+}=x P^{+}$. The quark fields carry a Dirac index, while flavor and color indices are suppressed. In order to ensure color gauge invariance of the correlator the Wilson-line

$$
\mathcal{W}\left(0, \xi^{-}\right)=\mathcal{P} \exp \left[-i g \int_{0}^{\xi^{-}} d \eta^{-} A^{+}\left(0, \eta^{-}, \overrightarrow{0}_{\perp}\right)\right]
$$

connecting the two quark fields is needed. It is well known that this gauge link encodes the re-interaction of the struck quark with the target system via the exchange of collinear gluons. In light-cone gauge $\left(A^{+}=0\right)$ the link disappears. The parton distributions can now be obtained from Eq. (1) using suitable projections. For instance, the unpolarized quark distribution is given by $f_{1}(x)=\operatorname{Tr}\left(\Phi \gamma^{+}\right) / 2$. 
In inclusive DIS only the longitudinal momentum fraction of the struck parton is fixed by external kinematics, while its transverse momentum cannot be measured but is rather integrated over. This situation changes in semi-inclusive DIS (like $e^{-} p \rightarrow e^{-} H X$ ), where in addition to the scattered electron a hadron $H$ is detected in the final state. If the cross section is kept differential in the transverse momentum $\vec{P}_{h \perp}$ of the hadron, one is sensitive to both the transverse momentum on the parton distribution side (transverse momentum of the quark relative to the target) and on the fragmentation side. At tree level, it is relatively easy to establish factorization into TMD parton distributions, TMD fragmentation functions and a hard scattering [23]. The Drell-Yan process (like $p p \rightarrow \mu^{+} \mu^{-} X$ ) with a low transverse momentum of the lepton-pair, and the reaction $e^{+} e^{-} \rightarrow H_{1} H_{2} X$, where the two detected hadrons in back-to-back jets have a low transverse momentum relative to each other, can be described within the same formalism of Ref. [23]. Once gluonic corrections are included it becomes quite complicated to establish QCD-factorization for this class of processes [24, 25, 26]. One source of the complications is the presence of soft gluons, which lead to an additional non-perturbative factor (soft factor) in a factorization formula. In the case of the one-loop calculations for T-odd correlation functions to be discussed below these difficulties don't show up yet. Therefore, we refrain from elaborating more on this issue here.

The correlator through which the TMD parton distributions are defined reads

$$
\Phi_{i j}\left(x, \vec{k}_{\perp}, S\right)=\left.\int \frac{d \xi^{-} d^{2} \vec{\xi}_{\perp}}{(2 \pi)^{3}} e^{i k \cdot \xi}\left\langle P, S\left|\bar{\psi}_{j}(0) \mathcal{W}(0, \xi) \psi_{i}(\xi)\right| P, S\right\rangle\right|_{\xi^{+}=0},
$$

where the parton distributions can again be obtained using suitable projections. It turns out that eight TMD parton distributions can appear at leading twist [27, 28], i.e., at leading order of a $1 / Q$-expansion of observables, where $Q$ denotes the hard external momentum of the process. In contrast to the correlation functions which show up in inclusive processes, with two quark fields separated only along one light-cone direction, the quark fields in Eq. (3) have also a separation in transverse position space. Moreover, for a proper definition of TMD parton distributions the gauge link in (3) must not connect the quark fields by the shortest line but rather has to take the form [29, 13, 30, 31]

$$
\mathcal{W}(0, \xi)_{D I S}=\left[0,0, \overrightarrow{0}_{\perp} ; 0, \infty, \overrightarrow{0}_{\perp}\right] \times\left[0, \infty, \overrightarrow{0}_{\perp} ; 0, \infty, \vec{\xi}_{\perp}\right] \times\left[0, \infty, \vec{\xi}_{\perp} ; 0, \xi^{-}, \vec{\xi}_{\perp}\right]
$$

In this equation, $\left[a^{+}, a^{-}, \vec{a}_{\perp} ; b^{+}, b^{-}, \vec{b}_{\perp}\right]$ denotes the Wilson line connecting the points $a^{\mu}=\left(a^{+}, a^{-}, \vec{a}_{\perp}\right)$ and $b^{\mu}=\left(b^{+}, b^{-}, \vec{b}_{\perp}\right)$ along a straight line. In covariant gauges like Feynman gauge the Wilson line at the light-cone infinity (second piece on the rhs in Eq. (4) can be neglected. However, this line has to be taken into account in light-cone gauge [30 31], because the transverse gluon potential doesn't vanish at the light-cone infinity.

It is now important to note that the path of the gauge link depends on the process. While for DIS the Wilson lines are future-pointing, they are past-pointing for Drell-Yan [13, 31, 17],

$$
\mathcal{W}(0, \xi)_{D Y}=\left[0,0, \overrightarrow{0}_{\perp} ; 0,-\infty, \overrightarrow{0}_{\perp}\right] \times\left[0,-\infty, \overrightarrow{0}_{\perp} ; 0,-\infty, \vec{\xi}_{\perp}\right] \times\left[0,-\infty, \vec{\xi}_{\perp} ; 0, \xi^{-}, \vec{\xi}_{\perp}\right] .
$$

As a consequence, the definitions of TMD parton distributions are different for both processes, i.e., there is a danger of non-universality for these objects. Exactly the same feature appears in the case of TMD fragmentation functions, which a priori have a different gauge link in semi-inclusive DIS compared to $e^{+} e^{-}$annihilation [17]. Whether and how one can still obtain universality for these TMD correlation functions will be discussed in the following two sections.

Two out of the eight leading twist TMD parton distributions are T-odd, and will be of particular importance for the subsequent discussion. The same holds for T-odd fragmentation functions. The following list (in the notation of Refs. [27, 28]) summarizes these functions and their meaning:

- $f_{1 T}^{\perp}$ : distribution of an unpolarized quark in a transversely polarized target [12]

- $h_{1}^{\perp}$ : distribution of a transversely polarized quark in an unpolarized target [28] 
- $D_{1 T}^{\perp}$ : fragmentation of an unpolarized quark into a transversely polarized hadron [27]

- $H_{1}^{\perp}$ : fragmentation of a transversely polarized quark into an unpolarized hadron [14]

T-odd correlation functions typically generate azimuthal/single spin asymmetries. For instance, in the case of the Sivers function $f_{1 T}^{\perp}$ one is dealing with a (T-odd) correlation $\vec{S}_{\perp} \cdot\left(\vec{P} \times \vec{k}_{\perp}\right)$ between the transverse target spin $\vec{S}_{\perp}$, the target momentum $\vec{P}$, and the transverse momentum of the quark $\vec{k}_{\perp}$. For a fixed target spin, this correlation gives rise to an azimuthal asymmetry of the quark distribution about the axis defined by the target momentum. In 1993, it was proved that T-odd parton distributions should vanish because of T-invariance of the strong interaction [14]. (This claim lateron needed to be revised as will be explained in Sect. 3]) In contrast, T-odd fragmentation functions can exist because of final state interactions in the fragmentation process. Such interactions can lead to a non-trivial phase (imaginary part) in the scattering amplitude, which is needed to obtain a finite T-odd asymmetry [14].

\section{Transverse single spin asymmetry in DIS}

We now turn the attention to possible observable effects of the Wilson line discussed in the previous section, i.e., effects due to the rescattering of the struck quark via the exchange of longitudinally polarized gluons. It has been demonstrated that even for inclusive DIS this rescattering influences the cross section [10]. To be specific, it yields a reduction of the cross section which can be interpreted as shadowing effect [10].

An observable being entirely connected with the gauge link has then been considered in Ref. [11]. In this work a simple model for a SSA with a transversely polarized spin- $\frac{1}{2}$ target has been constructed which will be discussed in some detail in this section. The relevant process is

$$
\gamma^{*}(q)+p(p, \lambda) \rightarrow q\left(p_{1}, \lambda^{\prime}\right)+s\left(p_{2}\right)
$$

and is shown in Fig. 11 The quark $q$ in the final state may either fragment into hadrons plus remnants or form a jet. For our purpose, however, it is not necessary to consider the hadronization of the quark. A simple spectator model with a scalar diquark spectator $s$ is used for the (proton) target [11]. In this model the proton has no electromagnetic charge, and a charge $e_{1}$ is assigned to the quark. The interaction between the proton, the quark and the spectator is given by a scalar vertex with the coupling constant $g$.

We treat the process (6) in the Breit frame of the virtual photon. (Note that in [11] a different reference frame was used.) The proton has a large plus-momentum $Q / x$, where $x=x_{B j}+\mathcal{O}\left(1 / Q^{2}\right)$. The quark carries the large minus-momentum $p_{1}^{-} \approx q^{-}$and a soft transverse momentum $\vec{\Delta}_{\perp}$. These requirements specify the kinematics:

$$
\begin{aligned}
& q=\left(-Q, Q, \overrightarrow{0}_{\perp}\right), \quad p=\left(\frac{Q}{x}, \frac{x M^{2}}{Q}, \overrightarrow{0}_{\perp}\right), \\
& p_{1}=\left(\frac{\vec{\Delta}_{\perp}^{2}+m_{q}^{2}}{Q}, Q, \vec{\Delta}_{\perp}\right), \quad p_{2}=\left(\frac{Q(1-x)}{x}, \frac{x\left(\vec{\Delta}_{\perp}^{2}+m_{s}^{2}\right)}{Q(1-x)},-\vec{\Delta}_{\perp}\right) .
\end{aligned}
$$

The expressions for $q$ and $p$ are exact, while for $p_{1}$ and $p_{2}$ just the leading terms have been listed. In particular, sometimes the $1 / Q^{2}$ corrections of $p_{1}^{-}$and $p_{2}^{+}$are needed which can be readily obtained from 4-momentum conservation.

In the Breit frame the leading twist contribution is given by the two transverse components $J^{1,2}$ of the electromagnetic current. (We define the current through the scattering amplitude via $T=\varepsilon_{\mu} J^{\mu}$, with $\varepsilon$ denoting the polarization vector of the virtual photon.) For our purpose it is sufficient to treat $J^{1}$, which 

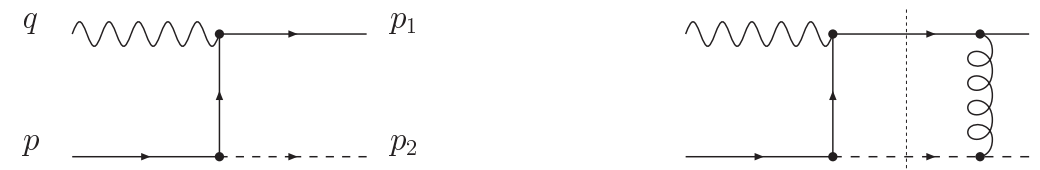

Fig. 1 Tree-level and relevant one-loop contribution for single spin asymmetry in DIS in spectator model (see also text). The spectator is indicated by a dashed line. The thin line characterizes the possible on-shell intermediate state.

reads for the tree-level diagram on the lhs in Fig. 1

$$
\begin{aligned}
J_{(0)}^{1}\left(\lambda, \lambda^{\prime}\right)= & e_{1} g \frac{1}{\left(p_{1}-q\right)^{2}-m_{q}^{2}} \bar{u}\left(p_{1}, \lambda^{\prime}\right) \gamma^{1}\left(p_{1} \cdot \gamma-q \cdot \gamma+m_{q}\right) u(p, \lambda) \\
= & -e_{1} g \frac{1-x}{\sqrt{x}\left|\vec{\Delta}_{\perp}\right|} \frac{Q}{\vec{\Delta}_{\perp}^{2}+\tilde{m}^{2}}\left[\left(\lambda m_{q}\left(M x+m_{q}\right)-\lambda\left(\Delta^{1}+i \lambda \Delta^{2}\right)^{2}\right) \delta_{\lambda,-\lambda^{\prime}}\right. \\
& \left.\quad+\left(\left(M x+m_{q}\right)\left(\Delta^{1}-i \lambda \Delta^{2}\right)+m_{q}\left(\Delta^{1}+i \lambda \Delta^{2}\right)\right) \delta_{\lambda, \lambda^{\prime}}\right], \\
& \text { with } \quad \tilde{m}^{2}=x(1-x)\left(-M^{2}+\frac{m_{q}^{2}}{x}+\frac{m_{s}^{2}}{1-x}\right) .
\end{aligned}
$$

The transverse SSA is given by the ratio $\sigma_{p o l} / \sigma_{u n p}$, where the unpolarized and the polarized (polarization along the $x$-axis) cross sections are computed according to

$$
\begin{aligned}
\sigma_{\text {unp }} & \propto \frac{1}{2} \sum_{\lambda, \lambda^{\prime}}\left(J^{1}\left(\lambda, \lambda^{\prime}\right)\right)^{\dagger} J^{1}\left(\lambda, \lambda^{\prime}\right), \\
\sigma_{\text {pol }} & \propto \frac{1}{2} \sum_{\lambda^{\prime}}\left[\left(J^{1}\left(s_{x}=\uparrow, \lambda^{\prime}\right)\right)^{\dagger} J^{1}\left(s_{x}=\uparrow, \lambda^{\prime}\right)-\left(J^{1}\left(s_{x}=\downarrow, \lambda^{\prime}\right)\right)^{\dagger} J^{1}\left(s_{x}=\downarrow, \lambda^{\prime}\right)\right] .
\end{aligned}
$$

A tree-level calculation taking only the expression in Eq. (8) into account leads to a vanishing asymmetry because the scattering amplitude has no imaginary part. In order to generate a finite imaginary part loop corrections have to be included, where we limit the analysis to the one-loop approximation. For simplicity, the gluon exchange is modelled by an Abelian gauge field [11]. It is easy to see that for the process in (6) only the box graph on the rhs in Fig. 11 which describes the re-interaction of the struck quark with the target system to lowest order, gives rise to an imaginary part. Now one indeed obtains a non-zero transverse SSA which is given by the interference of the tree-level amplitude with the imaginary part of the one-loop amplitude. Explicitly, one finds [11]

$$
A_{U T, x}=-\frac{\left(e_{1}\right)^{2}}{8 \pi} \frac{2\left(M x+m_{q}\right) \Delta^{2}}{\left(M x+m_{q}\right)^{2}+\vec{\Delta}_{\perp}^{2}} \frac{\vec{\Delta}_{\perp}^{2}+\tilde{m}^{2}}{\vec{\Delta}_{\perp}^{2}} \ln \frac{\vec{\Delta}_{\perp}^{2}+\tilde{m}^{2}}{\tilde{m}^{2}} .
$$

Obviously, the asymmetry vanishes if the transverse momentum of the quark vanishes. We emphasize again that the SSA would be zero if there were no re-interaction of the struck quark.

Soon after the publication of Ref. [11] it has been realized that the asymmetry in (11) is actually not a new effect, but can rather be understood as a model calculation for the T-odd Sivers function including its gauge link [13]. Therefore, the work in [11] has demonstrated for the first time explicitly that T-odd parton distributions can be non-zero. This result was at variance with the proof [14] according to which T-odd parton distributions should vanish because of T-invarince of the strong interaction. In fact it turned out that the proof no longer holds once the gauge link is taken into account [13]. 

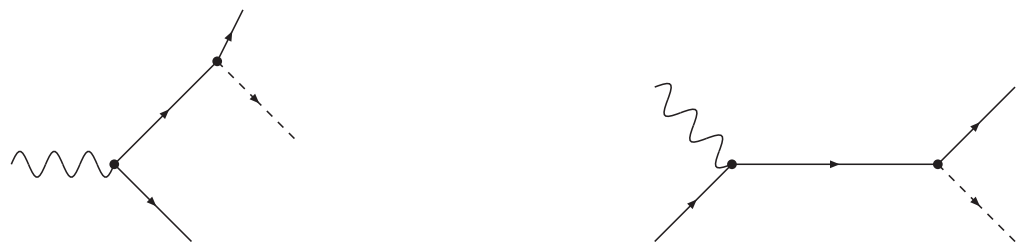

Fig. 2 Tree-level diagrams for fragmentation in $e^{+} e^{-}$annihilation and semi-inclusive DIS. In both cases a quark fragments into a spin- $\frac{1}{2}$ hadron and a scalar remnant (dashed line).

As mentioned in Sect. 2 the parton distributions in DIS and Drell-Yan have a different gauge link which endangers their universality. Nevertheless, the two definitions can still be related using time-reversal. It has been found that all six T-even TMD parton distributions are universal, while a violation of universality appears only for the two T-odd functions in the sense that they have a reversed sign in both processes [13],

$$
\begin{aligned}
\left.f_{1 T}^{\perp}\right|_{D I S} & =-\left.f_{1 T}^{\perp}\right|_{D Y}, \\
\left.h_{1}^{\perp}\right|_{D I S} & =-\left.h_{1}^{\perp}\right|_{D Y} .
\end{aligned}
$$

From a practical point of view this violation of universality has no direct consequence, since the possibility of relating cross sections of different processes is not spoiled. An experimental investigation of the relations (1213) would serve as a very important check of our present-day understanding of the nature of T-odd parton distributions and, hence, our understanding of SSA.

\section{Universality of fragmentation functions}

Now we want to investigate the influence of the Wilson line on the fragmentation process. Fragmentation functions in DIS and $e^{+} e^{-}$annihilation a priori have different Wilson lines (future-pointing for $e^{+} e^{-}$, past-pointing for DIS). However, in contrast to parton distributions, they cannot be related using timereversal because they involve a semi-inclusive sum over out-states [17] 18],

$$
\left.\sum_{X} \mid A, X, \text { out }\right\rangle\langle A, X, \text { out }|
$$

Time-reversal converts these into in-states, and therefore is useless in order to establish any relation between the fragmentation functions for both reactions. This means that fragmentation functions are nonuniversal, unless another argument comes to our aid. To investigate this point a model calculation for a transverse SSA in fragmentation (unpolarized quark into transversely polarized hadron, i.e., a model for $D_{1 T}^{\perp}$ ) has been performed [19], which is quite similar to the calculation discussed in the previous section.

In Fig. 2] the tree-level diagrams of the fragmentation in $e^{+} e^{-}$annihilation and in DIS are displayed. For $e^{+} e^{-}$annihilation we consider the decay of a timelike virtual photon into a $q \bar{q}$ pair, where the quark fragments into a spin- $\frac{1}{2}$ hadron (e.g. a proton) and a scalar remnant, i.e.,

$$
\gamma^{*} \rightarrow \bar{q}+p+s
$$

The fragmentation of the quark is described in the model of Ref. [11] used in Sect. 3 The one-loop corrections are shown in Fig. 3 For $e^{+} e^{-}$annihilation (semi-inclusive DIS) a single Abelian gluon is exchanged between the remnant and the antiquark (initial quark). These diagrams provide a simple model for the lowest order contribution of the gauge link of the fragmentation function. Two cuts (on-shell quark and antiquark, as well as on-shell antiquark and remnant) for $e^{+} e^{-}$annihilation have no counterpart in semi-inclusive DIS. The quark-photon cut in $e^{+} e^{-}$annihilation corresponds to the cut in DIS. Note that 

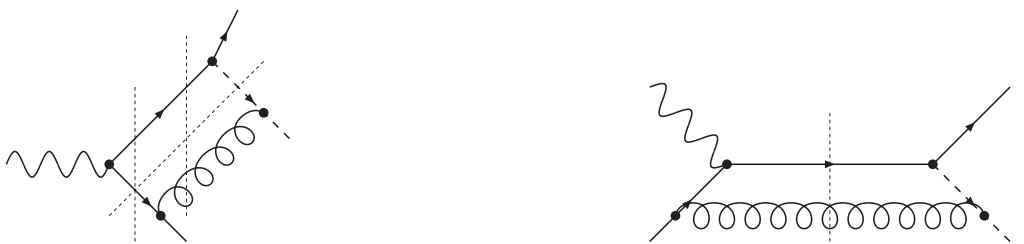

Fig. 3 One-loop diagrams for fragmentation in $e^{+} e^{-}$annihilation and semi-inclusive DIS. The possible on-shell intermediate states are indicated by thin lines.

the one-loop correction at the photon vertex, which is also related to the gauge link of the fragmentation function, is irrelevant for a transverse SSA, i.e., it neither contributes to $D_{1 T}^{\perp}$ nor to $H_{1}^{\perp}$. All remaining one-loop graphs have no relation to the gauge link. These effects are obviously universal (see, e.g., also Ref. [32]), and won't be considered in the following.

The calculation for the transverse SSA is similar to the case of the target asymmetry considered in Sect. 3 Here we just focus on the main results, while detailed formulae can be found in Ref. [19]. We first consider the two on-shell intermediate states in $e^{+} e^{-}$annihilation which have no counterpart in DIS and, hence, form a potential source of non-universality of the transverse SSA. However, it turns out that the contributions of both intermediate states to the SSA cancel each other [19],

$$
\left.\mathcal{A}^{\bar{q} q}\right|_{e^{+} e^{-}}=-\left.\mathcal{A}^{\bar{q} s}\right|_{e^{+} e^{-}} .
$$

This cancellation is exact for the leading twist term of the asymmetry, which is sufficient for our investigation here. At subleading twist the equality 16 breaks down.

One is now left with the on-shell $q g$ intermediate state only. By explicit calculation it has been shown that the corresponding contribution to the SSA coincides for both processes [19],

$$
\left.\mathcal{A}^{q g}\right|_{D I S}=\left.\mathcal{A}^{q g}\right|_{e^{+} e^{-}}
$$

i.e., the asymmetry in both processes has the same sign. In particular, one does not observe a sign-reversal for the one-loop box graph that is associated with the gauge link of the fragmentation function. The origin for the different behaviour compared to the T-odd target asymmetry is directly related to the different kinematics one is dealing with in fragmentation. Note for instance that, in contrast to the case of the target asymmetry, in fragmentation the surviving contribution to the SSA involves a cut of the gauge boson propagator.

Altogether, one finds that the total transverse SSA in $e^{+} e^{-}$annihilation and in semi-inclusive DIS are equal, i.e, the T-odd fragmentation of an unpolarized quark into a transversely polarized spin- $\frac{1}{2}$ hadron is universal in the one-loop model. This implies universality of the fragmentation function $D_{1 T}^{\perp}$. The same conclusion holds for the Collins function as well [19]. Therefore, we have the results

$$
\begin{aligned}
\left.D_{1 T}^{\perp}\right|_{D I S} & =\left.D_{1 T}^{\perp}\right|_{e^{+} e^{-}}, \\
\left.H_{1}^{\perp}\right|_{D I S} & =\left.H_{1}^{\perp}\right|_{e^{+} e^{-}} .
\end{aligned}
$$

This model calculation has indicated that fragmentation functions might well be universal despite the $a$ priori reversed Wilson lines they have in $e^{+} e^{-}$annihilation compared to semi-inclusive DIS. Recently, it has indeed been shown by a more general analysis that this suspicion is true [18]. Universality not only holds for the two T-odd fragmentation functions but also for the T-even ones. Moreover, it has been argued that it should be valid to all orders in perturbation theory. The crucial observation is that, due to the special analytical structure of the fragmentation process, one is not sensitive to the direction of the Wilson lines. In particular, factorization for semi-inclusive DIS can be derived with both past-pointing and future-pointing Wilson lines for the fragmentation functions [18]. 

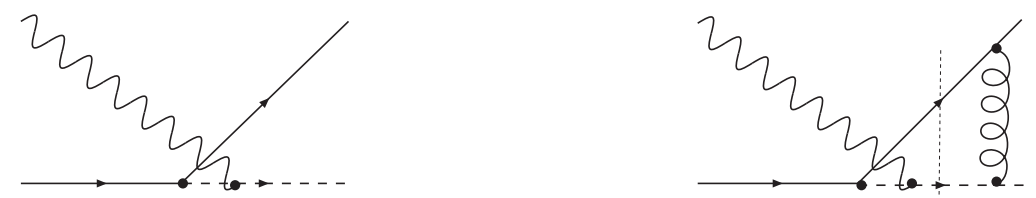

Fig. 4 Tree-level and one-loop contribution to the process in 6. Such diagrams, where the photon couples to the spectator, are needed in order to ensure electromagnetic gauge invariance at subleading twist.

\section{Longitudinal single spin asymmetries in DIS}

The transverse SSA $A_{U T}$ in semi-inclusive DIS discussed in Sect. 3 is a leading twist observable. One may wonder whether also the subleading asymmetries $A_{U L}$ (longitudinally polarized target) and $A_{L U}$ (longitudinally polarized lepton) are affected by the rescattering of the struck quark. It is quite important to clarify this issue, because most of the existing data and of the analyses (see e.g. Refs. [33, 34, 35, 36 37, 38, 39, 40|) are concerned with these twist-3 observables.

In most of the theoretical papers a formalism is used which only includes T-odd fragmentation functions [41, 27]. In this scenario one finds schematically $A_{L U} \propto e H_{1}^{\perp}$ and $A_{U L} \propto h_{L} H_{1}^{\perp}$, with the twist-3 T-even parton distributions $e$ and $h_{L}$. The most recent works in Refs. [39, 40] on the beam spin asymmetry extended the analysis by considering also T-odd parton distributions. This leads for $A_{L U}$ to an additional term $h_{1}^{\perp} E$, where $E$ is a twist-3 fragmentation function. However, it was still unclear if also possible higher twist T-odd distribution functions, generated by the same rescattering mechanism that leads to twist-2 T-odd functions, could be relevant for the longitudinal SSA.

In order to proceed at this point, the spectator model calculation for $A_{U T}$ in Ref. [11] was extended to the twist-3 case [20]. In this work, $A_{L U}$ was computed from the Feynman diagrams in Fig. 11 and a non-zero result was obtained. But it turned out that, at the twist-3 level, the graphs in Fig. 11 no longer satisfy electromagnetic gauge invariance. This has been corrected in Ref. [21] by including the diagrams in Fig. 4 where the photon couples to the diquark spectator. Moreover, also the target spin asymmetry $A_{U L}$ has been computed in [21]. The gauge invariant treatment yields non-zero results for both asymmetries,

$$
A_{L U} \neq 0, \quad A_{U L} \neq 0
$$

where details of the calculation can be found in [21].

Let us now turn to the implications of this calculation. First of all, it is obvious that the diagrams in Fig. (4) are not compatible with the parton model, and therefore are also not compatible with factorization. However, this does not necessarily mean that factorization for semi-inclusive DIS is broken at subleading twist, even if it has not yet been proved. One rather reaches the limitations of the perturbative spectator model for the nucleon. In particular, one finds that large momentum transfers at the proton-quark-diquark vertex, which appear in the graphs in Fig. 4 are not suppressed. Actually they get enhanced, which is at variance with the parton model.

Despite this shortcoming of the spectator model, it has been argued that in a parton model description of $A_{L U}$ and $A_{L U}$ subleading T-odd distributions should be present [21]. Mainly for two reasons this speculation has been made: first, within the model calculation non-zero asymmetries arise already from the diagrams in Fig. 11 whose kinematics is compatible with the parton model. Second, there is no reason why the asymmetries should not contain higher order T-odd distribution functions.

A revised parton model analysis for $A_{L U}$ and $A_{U L}$ lateron has confirmed this speculation [22]. Both asymmetries contain an additional term with a twist-3 T-odd distribution function, which have not been taken into account in the literature before. The main observation has been that additional functions are generated once the gauge link in the correlator in Eq. (3) is taken into account [42, 22]. 


\section{Conclusions}

We have summarized important recent progress in the understanding of single spin asymmetries in hard scattering processes. All these developments are based on the fact that collinear gluon exchange (e.g., in DIS between the struck quark and the target system) leads to observable effects. In a factorized description these contributions are encoded in the gauge link of parton distributions and fragmentation functions.

The most striking influence of collinear gluons appears in the case of single spin asymmetries/T-odd correlation functions. While T-odd fragmentation functions are non-zero without such gluon exchange, Todd parton distributions vanish if their gauge link is neglected. The existence of T-odd parton distributions considerably enriches the phenomenology of single spin asymmetries. For instance, in semi-inclusive DIS such effects not only enter the leading twist asymmetry $A_{U T}$, but also the twist-3 observables $A_{U L}$ and $A_{L U}$. Since the gauge link is process dependent, it endangers the universality of transverse momentum dependent correlation functions. However, it has been shown that the only violation of universality appears for T-odd parton distributions in terms of a reversed sign between DIS and the Drell-Yan process.

Eventually, our short review nicely shows that model calculations quite often lead to the discovery of new model-independent results. This remains true despite the limitations models typically have.

Acknowledgements We are very grateful to Klaus Goeke for the pleasant and stimulating atmosphere at the Institut fuer Theoretische Physik II, Ruhr-Universität Bochum. One of us (A.M.) would like to thank John Collins for fruitful collaboration. The work has been partly supported by the DFG, and the Sofia Kovalevskaya Programme of the Alexander von Humboldt Foundation.

\section{References}

[1] D. L. Adams et al., Phys. Lett B 264, 462 (1991).

[2] A. V. Efremov and O. V. Teryaev, Phys. Lett. B 150, 383 (1985).

[3] J. Qiu and G. Sterman, Phys. Rev. Lett. 67, 2264 (1991).

[4] J. Qiu and G. Sterman, Phys. Rev. D 59, 014004 (1999).

[5] P. G. Ratcliffe, Eur. Phys. J. C8, 403 (1999).

[6] M. Anselmino, M. Boglione, U. D’Alesio, E. Leader, and F. Murgia, hep-ph/0408356

[7] HERMES Collaboration, A. Airapetian, et al., Phys. Rev. Lett. 84, 4047 (2000); Phys. Rev. D 64, 097101 (2001); Phys. Lett. B 562, 182 (2003); hep-ex/0408013

[8] CLAS Collaboration, H. Avakian, et al., Phys. Rev. D 69112004 (2004).

[9] G. L. Kane, J. Pumplin, and W. Repko, Phys. Rev. Lett. 41, 1689 (1978).

[10] S. J. Brodsky, P. Hoyer, N. Marchal, S. Peigné, and F. Sannino, Phys. Rev. D 65, 114025 (2002).

[11] S. J. Brodsky, D. S. Hwang, and I. Schmidt, Phys. Lett. B 530, 99 (2002).

[12] D. W. Sivers, Phys. Rev. D 41, 83 (1990); Phys. Rev. D 43, 261 (1991).

[13] J. C. Collins, Phys. Lett. B 536, 43 (2002).

[14] J. C. Collins, Nucl. Phys. B396, 161 (1993).

[15] J. C. Collins and G. A. Ladinsky, hep-ph/9411444

[16] A. Bacchetta, R. Kundu, A. Metz, and P. J. Mulders, Phys. Lett. B 506, 155 (2001).

[17] D. Boer, P. J. Mulders, and F. Pijlman, Nucl. Phys. B667, 201 (2003).

[18] J. C. Collins and A. Metz, hep-ph/0408249

[19] A. Metz, Phys. Lett. B 549, 139 (2002).

[20] A. Afanasev and C. E. Carlson, hep-ph/0308163

[21] A. Metz and M. Schlegel, hep-ph/0403182 to appear in EPJA.

[22] A. Bacchetta, P. J. Mulders, and F. Pijlman, Phys. Lett. B 595, 309 (2004).

[23] J. P. Ralston and D. E. Soper, Nucl. Phys. B152, 109 (1979).

[24] J. C. Collins and D. E. Soper, Nucl. Phys. B193, 381 (1981); Nucl. Phys. B213, 545 (1983) (E).

[25] X. Ji, J. Ma, and F. Yuan, hep-ph/0404183

[26] X. Ji, J. Ma, and F. Yuan, Phys. Lett. B 597, 299 (2004).

[27] P. J. Mulders and R. D. Tangerman, Nucl. Phys. B461, 197 (1996); Nucl. Phys. B484, 538 (1997) (E).

[28] D. Boer and P. J. Mulders, Phys. Rev. D 57, 5780 (1998).

[29] J. C. Collins and D. E. Soper, Nucl. Phys. B194, 445 (1982). 
[30] X. Ji and F. Yuan, Phys. Lett. B 543, 66 (2002).

[31] A. V. Belitsky, X. Ji, and F. Yuan, Nucl. Phys B656, 165 (2003).

[32] O. V. Teryaev, Czech. J. Phys. 53, 47A (2003).

[33] K. A. Oganessyan, H. R. Avakian, N. Bianchi, and A. M. Kotzinian, hep-ph/9808368

[34] A. V. Efremov, K. Goeke, M. V. Polyakov, and D. Urbano, Phys. Lett. B 478, 94 (2000).

[35] E. De Sanctis, W. D. Nowak, and K. A. Oganessyan, Phys. Lett. B 483, 69 (2000).

[36] B. Q. Ma, I. Schmidt, and J. J. Yang, Phys. Rev. D 63, 037501 (2001).

[37] A. V. Efremov, K. Goeke, and P. Schweitzer, Phys. Lett. B 522, 37 (2001); Phys. Lett. B 544, 389 (2002) (E).

[38] A. V. Efremov, K. Goeke, and P. Schweitzer, Phys. Rev. D 67, 114014 (2003).

[39] F. Yuan, Phys. Lett. B 589, 28 (2004).

[40] L. P. Gamberg, D. S. Hwang, and K. A. Oganessyan, Phys. Lett. B 584, 276 (2004).

[41] J. Levelt and P. J. Mulders, Phys. Lett. B 338, 357 (1994).

[42] K. Goeke, A. Metz, P. V. Pobylitsa, and M. V. Polyakov, Phys. Lett. B 567, 23 (2003). 